\title{
CAMBIO CLIMÁTICO: IMPACTOS Y PERSPECTIVAS DE INVESTIGACIÓN DESDE UNA VISIÓN MULTIDISCIPLINAR ${ }^{1}$
}

\author{
CLIMATE CHANGE: IMPACTS AND PERSPECTIVES OF RESEARCH FROM A \\ MULTIDISCIPLINARY VISION
}

\section{MUDANÇA CLIMÁTICA: IMPACTOS E PERSPECTIVAS DE INVESTIGAÇÃO DESDE UMA VISÃO MULTIDICIPLINAR}

\section{Por: FORERO CANTOR_ Germán Augusto, SALDARRIAGA MUÑOZ_ Juan Pablo, VARGAS ROMERO_ Melissa}

Magister en Economía de la Universidad de los Andes, Economista Universidad Nacional. Profesor Asistente adscrito al Departamento de Economía y Finanzas de la Universidad del Tolima. Email: gaforeroc@ut.edu.co,Colombia.

Magister en Economía y Economista de la Universidad de Antioquia. Profesor Asistente adscrito al Departamento de Economía y Finanzas de la Universidad del Tolima. Email: jpsaldarriagam@ut.edu. co, Colombia.

Economista de la Universidad del Tolima. Investigadora del Grupo de Investigación en Economía de la Universidad del Tolima - GRIECONUT. Email: mvargasro@ut.edu.co, Colombia.

Recibido: 6 de septiembre de 2016

Aprobación definitiva: 8 de junio de 2017

DOI: http://dx.doi.org/10.22267/rtend.171802.80

\begin{abstract}
RESUMEN
La preocupación que en los últimos años ha surgido por parte de los países desarrollados ante los crecientes impactos económicos generados por el Cambio Climático el cual se refleja en el incremento del número de investigaciones en este campo. La mayoría de los estudios muestran que el Cambio Climático afecta múltiples aspectos del entorno trayendo como resultado impactos directos sobre la economía de un país que en la mayoría de los casos se evidencia en alteraciones positivas y negativas del PIB. Los mayores impactos se presentan en los países del trópico donde paradójicamente hay una cantidad relativamente baja de investigaciones que
\end{abstract}

1 Este artículo surge como resultado del proyecto de investigación “Modelo de Gestión de Riesgo Empresarial como herramienta de mitigación para los impactos del Cambio Climático en empresas de producción de arroz en el departamento del Tolima" financiado por la Dirección de investigaciones de la Universidad del Tolima. 
aborden a profundidad el tema, siendo una posible causa de esto el desconocimiento que se tiene de los conceptos fundamentales relacionados con el tema. Este trabajo muestra desde una revisión actualizada y exhaustiva de la literatura un marco conceptual general que sirve como base para emprender procesos de investigación desde una perspectiva multidisciplinar alrededor de los principales impactos del Cambio Climático.

Palabras Clave: valoración de efectos ambientales, clima, calentamiento global.

JEL: Q51, Q54.

\begin{abstract}
In the past few years has arisen a concern about the growing impacts of the Climate Change in developed countries, which is reflected in the increased number of research in this field. Most studies show that climate change affects multiple aspects of the environment resulting in direct impacts on the economy of a country that in the majority of cases is evident in positive and negative changes of GDP. The greatest impacts occur in the tropical countries where paradoxically there are a number relatively low of research that address the topic, being a possible cause of this lack of knowledge that makes the fundamental concepts related to the topic at depth. Given the above, this work shows a general conceptual framework that serves as a base for research from a multidisciplinary perspective around the main impacts of climate change from a thorough and up-to-date review of the literature.
\end{abstract}

Keywords: valuation of environmental changes, climate, global warming

JEL: Q51, Q54.

\title{
RESUMO
}

A preocupação que nos últimos anos tem surgido por parte dos países desenvolvidos ante os crescentes impactos económicos gerados pela Mudança Climática o qual se reflete no incremento do número de investigações neste campo. A maioria dos estudos mostram que a Mudança Climática afecta múltiplos aspectos do meio trazendo como resultado impactos diretos sobre a economia de um país que na maioria dos casos se evidência em alterações positivas e negativas do PIB. Os maiores impactos apresentam-se nos países do trópico onde paradoxalmente há uma quantidade relativamente baixa de investigações que abordem a profundidade o tema, sendo uma possível causa disto o desconhecimento que se tem dos conceitos fundamentais relacionados com o tema. Este trabalho mostra desde uma revisão actualizada e exhaustiva da literatura um marco conceptual general que serve como base para empreender processos de investigação desde uma perspectiva multidiciplinar ao redor dos principais impactos da Mudança Climática.

Palavras-chave: valoração de efeitos ambientais, clima, aquecimento global.

JEL: Q51, Q54. 


\section{INTRODUCCIÓN}

Dada la dinámica del Cambio Climático en los últimos años las investigaciones relacionadas con este han dejado de ser de objeto de estudio exclusivo de académicos en el área de la meteorología y/o el clima. Esto se refleja al realizar una revisión de la literatura sobre el tema, la cual muestra que los impactos generados por el Cambio Climático son crecientes y se visibilizan principalmente a través de alteraciones en variables relacionadas con la producción agrícola, la disponibilidad de agua, los ecosistemas, la salud humana, el nivel de los océanos, etc., que alteran de manera directa a la economía en su conjunto.

Aunque parece haber un consenso generalizado por parte de algunos investigadores en cuanto a la necesidad de estudiar a profundidad las diversas relaciones existentes entre Cambio Climático y Economía -fundamentalmente los impactos del primero sobre la segunda- en algunos casos se siguen presentando algunas confusiones conceptuales al momento de abordar procesos de investigación relacionados con el tema, lo que de alguna forma ha limitado su desarrollo.

El objetivo del presente artículo es mostrar algunos elementos conceptuales básicos alrededor del Cambio Climático así como de posibles perspectivas de investigación que pretendan evaluar los impactos de este sobre la economía. El artículo consta de esta introducción que es la primera parte; en la segunda se muestran diferentes concepciones de lo que se entiende por cambio climático; en la cuarta se plantean algunos impactos que genera el cambio climático; en la quinta se muestran algunas líneas de trabajo en cuanto a impactos del cambio climático sobre la economía para finalmente terminar con unas conclusiones.

\section{CONCEPTUALIZANDO EL CAMBIO CLIMÁTICO}

El término clima proviene del griego klima que define inclinación y en los antiguos griegos hacía referencia a las diferentes inclinaciones que tenían los rayos del sol con la superficie terrestre. Actualmente, el clima se define como las condiciones típicas del estado del tiempo para un área dada (Mokhov and Eliseev, 2008). El sistema climático de nuestro planeta integra cinco subsistemas que son la atmósfera, los océanos, la biósfera, la superficie terrestre y la criósfera (Bradley,2015). Las múltiples interacciones que se presentan entre estos y que determinan el clima de nuestro planeta son muy complejas por lo que su estudio ha resultado complejo para los académicos expertos en el tema. En la tabla 1 se muestran algunos elementos propios de cada subsistema que inciden sobre las variables climáticas en el planeta ${ }^{2}$. A partir de esto, el clima se puede definir como una descripción estadística en términos de variabilidades y medias de variables como la temperatura, las precipitaciones y el viento que afectan al sistema climático en su conjunto.

2 Esta es una muestra muy pequeña de impactos generados por los subsistemas; un número más amplio de otras interacciones se puede encontrar en la bibliografía citada. 
Tabla 1. Componentes de Sistema Climático y algunas incidencias

\begin{tabular}{|c|c|}
\hline \multicolumn{2}{|r|}{ SISTEMA CLIMÁTICO } \\
\hline Subsistema & Característica principal \\
\hline Atmósfera & $\begin{array}{l}\text { Es el subsistema más variable. Atrapa y reirradia la energía a } \\
\text { otros componentes del sistema como los gases de invernadero } \\
\text { emitidos por la tierra y los océanos. }\end{array}$ \\
\hline Océanos & $\begin{array}{l}\text { Responde a influencias externas más lentamente que la } \\
\text { atmósfera. Absorbe C02 reduciendo su concentración en la } \\
\text { atmósfera }\end{array}$ \\
\hline Biósfera (Plantas y animales) & $\begin{array}{l}\text { La vegetación funciona como un agente retroalimentador } \\
\text { biogeoquímico y biogeofísico con la atmósfera. Influye sobre la } \\
\text { composición atmosférica a través de la remoción de dióxido de } \\
\text { carbono y la producción de aerosoles y oxigeno. }\end{array}$ \\
\hline Superficie Terrestre & \\
\hline $\begin{array}{l}\text { Criósfera (glaciales, niveve } \\
\text { estacional, hielo oceánico, }\end{array}$ & $\begin{array}{l}\text { Sus propiedades reflectivas marcan diferencias en la forma en } \\
\text { que se calienta el planeta }\end{array}$ \\
\hline
\end{tabular}

Fuente: Elaboración propia a partir de (Bradley, 2015;Hannah,20015)

Los estudios alrededor del Clima y el Cambio Climático (CC) no son algo novedoso, tal y como lo muestran (Weart, 2015 y Fleming, 1998). En su exposición, estos autores muestran que desde el siglo XIX ya se venían realizando trabajos alrededor del tema, destacando -entre otros- los trabajos de Joseph Fourier en 1824 quién encontró que las alteraciones que se presentaban en el clima estaban fundamentadas por cambios en la atmósfera, seguido por John Tyndall en 1859 quien pudo establecer que el dióxido de carbono y el metano eran gases que intervenían en el proceso de elevación de la temperatura y luego Svante Arrhenius en 1896 descubrió que la quema de combustibles fósiles en la tierra generaban dióxido de carbono, el cual influía de manera importante sobre el clima ${ }^{3}$.

En cuanto al concepto de Cambio Climático, existen diferentes acepciones que generan igual o mayor número de confusiones. Una de estas es que no se tiene clara la diferencia que existe entre el concepto de variabilidad climática y Cambio Climático. El primero hace referencia a alteraciones alrededor del promedio del valor que tome la variable climática que se establezca como referencia (temperatura por ejemplo) a través del tiempo -osea que siempre va a existir-, mientras que el segundo denota que para que exista Cambio Climático la variable climática utilizada debe presentar un comportamiento no estacionario, donde las fluctuaciones que se dan en la variable no tienen una media constante (Burroughs,2007). Los dos paneles que se muestran en la figura 1 exponen algunas situaciones de evolución de la temperatura a través del tiempo en las que se puede evidenciar tanto presencia como ausencia de Cambio Climático. En la serie (a) de ambos paneles aunque existe variación de la temperatura esta se da alrededor de una media que es estable a través del tiempo (primera condición de estacionariedad) mientras que en

3 Aquí se mencionan solo algunos eventos del siglo XIX. Para una descripción de eventos y trabajos desarrollados durante el siglo XX se pueden Consultar (Weart, 2015 y Fleming, 1998). 
las situaciones (b), (c) y (d) hay alteraciones en la tendencia de la temperatura por lo que en éstas si se evidencia el Cambio Climático.

\section{Figura 1. Series temporales de temperatura para analizar el cambio climático}
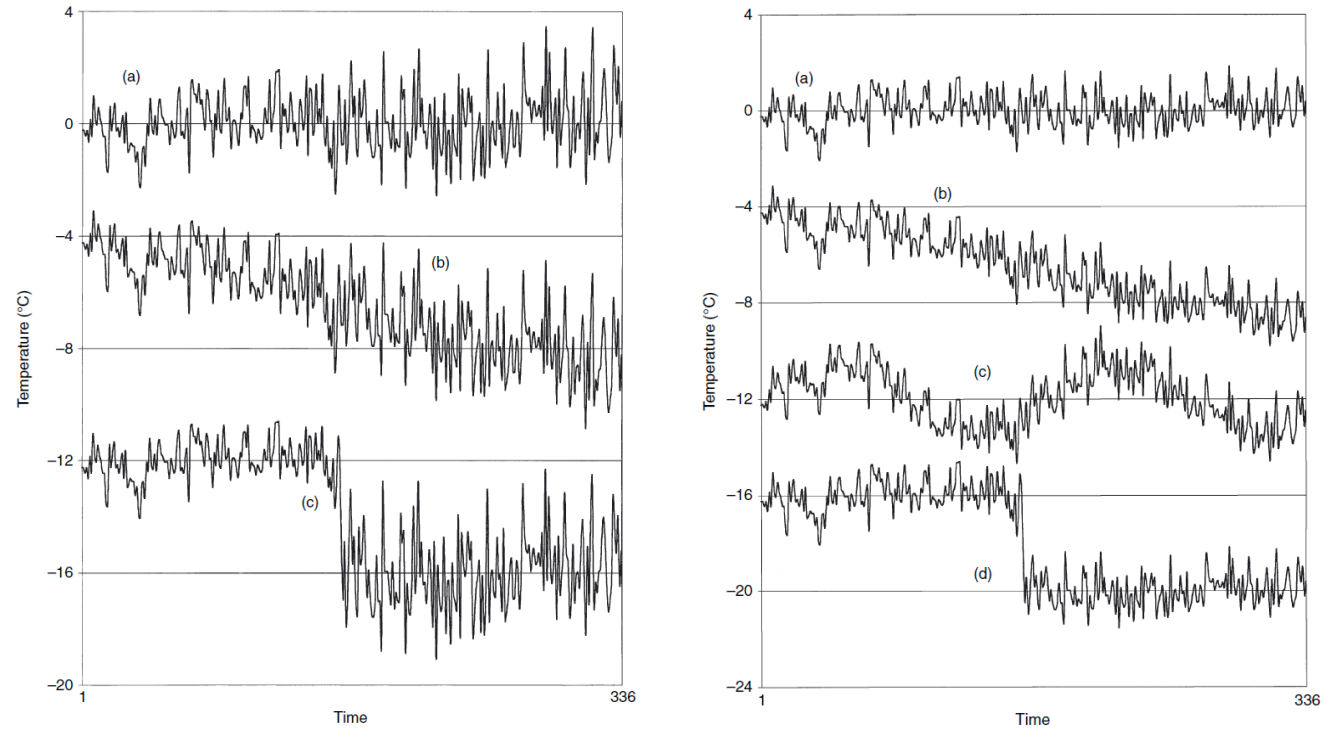

Fuente: Adaptación de los autores a partir de Burroughs (2007).

De otro lado, un viejo debate académico que aún persiste al momento de definir Cambio Climático tiene que ver con el hecho de sí en la definición se deben incluir o no las acciones antropogénicas como determinantes del mismo. Este debate puede resumirse en dos aproximaciones al concepto: una que podríamos denominar "clásica" y otra "integral". La primera, planteada por quienes no consideran la inclusión del componente antropogénico, establece que el Cambio Climático se define como las alteraciones que se pueden presentar en diversas variables meteorológicas, fundamentalmente por alteraciones naturales en los componentes del sistema climático, durante un período de tiempo determinado. La definición "integral" se extiende un poco más y agrega a la primera la acción del hombre como un factor que altera de manera directa el clima a través de las emisiones generadas por los combustibles fósiles lo que a su vez impacta a los componentes del sistema climático, especialmente a la atmósfera, en lo que la ONU denomina un período de tiempo comparable (Naciones Unidas, 1992); es decir, el Cambio Climático tiene como causa un componente natural que a su vez se ve complementado con un componente antropogénico ${ }^{4}$.

4 Dentro de los componentes naturales se tienen los gases de la atmósfera, la radiación solar, los rayos cósmicos, la actividad volcánica y las características de la órbita terrestre. Para estudiar con mayor profundidad estos factores se puede consultar Letcher (2009). 
Las anteriores definiciones se pueden complementar con las estrategias de medición para establecer la existencia de Cambio Climático. En este sentido una de las más utilizadas es el análisis de las denominadas anomalías de temperatura, donde estas se definen como un cambio a partir de un punto de referencia promedio de largo plazo ${ }^{5}$. Una anomalía positiva es un valor más caliente y una anomalía negativa es un valor más frío de temperatura. A manera de ejemplo, la figura 2 muestra valores de anomalía de temperatura para el período 1880-2004 en la tierra, encontrando que a partir de la década de los 70s las anomalías exhiben un incremento permanente lo cual ha sido tomado por la mayoría de los científicos como evidencia del Cambio Climático permanente a partir de dicha década.

Figura 2. Anomalías de temperatura en el planeta (1881-2004)

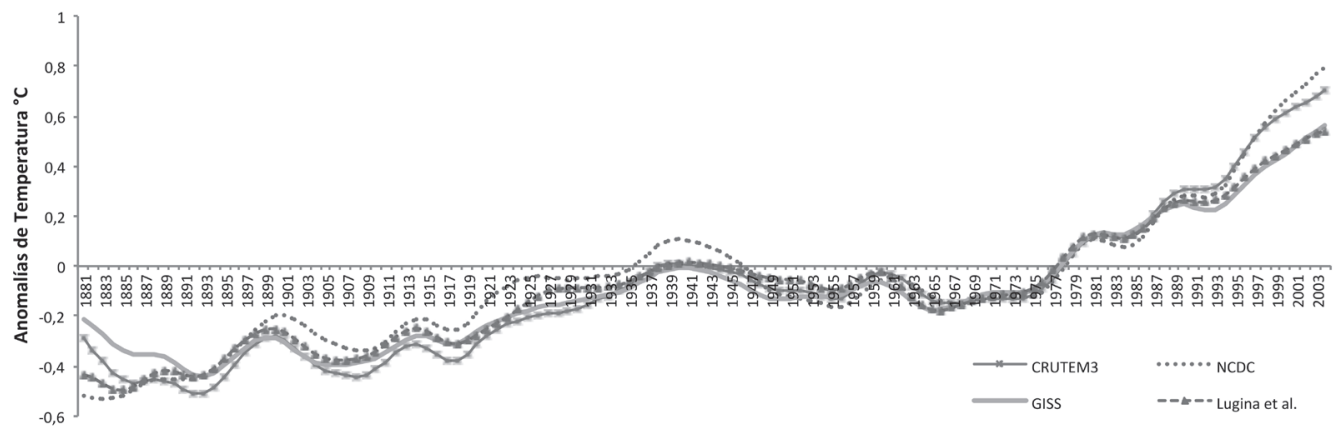

Fuente: construcción propia a partir de datos del Community Data Portal (CDP). Los datos utilizados son generados por el National Climate Data Center (NCDC), Goddard Institute for Space Studies (GISS), Climate Research Unit de la Universidad de East Anglia (de donde proviene el CRUTEM3) y Lugina et al. del Carbon Dioxide Information Analysis Center.

Aunque en la actualidad parece haber consenso en lo referente a la existencia de Cambio Climático este no es del todo completo. Bajo esta perspectiva vale anotar que la tierra en su conjunto sigue siendo un sistema complejo e imperfectamente entendido (Letcher,2009) el cual para ser analizado en términos climáticos requiere no solamente de análisis de nuestra historia reciente que podríamos denominar de corto plazo sino de la evolución climática de la tierra desde su misma formación en lo que se podría denominar un escenario de largo plazo.

La Tabla 1 muestra una escala geológica y cronológica de la tierra desde su formación hace alrededor de hace 4.6 billones de años hasta la actualidad y algunos eventos relacionados con el clima. Inicialmente al observar algunos eventos climáticos asociados a las eras se encuentra que en el Precámbrico y el Paleozoico las temperaturas eran bajas, efecto que se revirtió en el Mesozoico donde la temperatura muestra un incremento. Luego en el Cenozóico que abarca un período de tiempo desde hace 65 millones de años hasta el presente se visualizan fluctuaciones en la temperatura de la tierra, donde al hacer un

5 En general se utiliza como valor de referencia el promedio de temperatura para el período 1961-1990 ya que para este existe una certeza en la calidad y cantidad de las mediciones desarrolladas. 
Cambio climático: Impactos y perspectivas de investigación desde una visión multidisciplinar

análisis por épocas se encuentra que en el Pleistoceno la temperatura de la tierra era $5^{\circ} \mathrm{C}$ más baja que la que tenemos actualmente para luego en el Holeoceno volver a presentar un calentamiento que osciló entre $0.5^{\circ} \mathrm{C}$ y $2^{\circ} \mathrm{C}$. En nuestra historia más reciente (último milenio) se puede hablar de un calentamiento permanente desde mediados del siglo XVII hasta el presente, con un mayor impacto desde 1970 en donde se establece que la elevación de la temperatura adquiere un carácter monotónico.

\section{Tabla 1. Tabla Geocronológica climática}

\begin{tabular}{|c|c|c|c|}
\hline Era & Período & Epoca & Observaciones \\
\hline \multirow{7}{*}{ Cenozoico } & \multirow{4}{*}{ Cuaternario } & Últimos 150 años & $\begin{array}{l}\text { Incremento sustancial en la } \\
\text { incidencia antropogénica. Aumento } \\
\text { del dióxido de carbono de } 275-288 \\
\text { ppmv en el } 2012 \text {; el metano ha } \\
\text { pasado de } 830 \text { ppbv a } 1760 \text { ppbv en } \\
\text { el } 2000 \text {. Incremento en la radiación } \\
\text { solar. En el siglo XXI se presentaron } \\
\text { oscilaciones en el clima pero el } \\
\text { incremento en temperaturas se ha } \\
\text { dado monotónicamente desde } 1970 \text { s. }\end{array}$ \\
\hline & & $\begin{array}{l}\text { Ultimo milenio (Era } \\
\text { preindustrial) }\end{array}$ & $\begin{array}{l}\text { En el siglo XVII la temperatura } \\
\text { promedio fue } 0.5^{\circ} \mathrm{C} \text { menor que la del } \\
\text { siglo XIX y se ha incremento } \\
\text { gradualmente hasta mediados del } \\
\text { siglo XX }\end{array}$ \\
\hline & & Holeoceno (11.000 a-P) & $\begin{array}{l}\text { Se presentó un calentamiento } \\
\text { promedio que oscila entre } 0.5^{\circ} \mathrm{C}-2^{\circ} \mathrm{C}\end{array}$ \\
\hline & & $\begin{array}{c}\text { Pleistóceno (1.8Ma- } \\
11.000 \mathrm{a})\end{array}$ & $\begin{array}{l}\text { Se presentaron glaciaciones e } \\
\text { interglaciaciones. La temperatura era } \\
5^{\circ} \mathrm{C} \text { menor que la actual; el } \\
\text { enfriamiento relativo con respecto a } \\
\text { la era preindustrial era de entre } 3^{\circ} \mathrm{y} \\
4^{\circ} \mathrm{C} \text {. }\end{array}$ \\
\hline & \multirow{3}{*}{ Terciario (67Ma - 1.8MaAP) } & Plioceno (9-1.8 MaAP) & \\
\hline & & $\begin{array}{c}\text { Mioceno (25 - } 9 \text { MaAP) } \\
\text { Oligoceno (37-25 MaAP } \\
\text { Eoceno(56-37 MaAP) }\end{array}$ & \\
\hline & & Paleoceno(67-56 MaAP) & \\
\hline \multirow{3}{*}{ Mesozoico (236-67 MaAP) } & Cretácico (133 - 67 MaAP) & & Extremadamente caliente, $10-15^{\circ} \mathrm{C}$ \\
\hline & Jurásico (186-133 MaAP) & & $\begin{array}{l}\text { diferencia del clima entre los polos y } \\
\text { la zona ecuatorial era de } 15^{\circ} \mathrm{C} \text {, }\end{array}$ \\
\hline & Triásico (236-186MaAP) & & $\begin{array}{l}\text { actualmente es de } 46^{\circ} \mathrm{C} \text {. No hay } \\
\text { evidencia de hielo bajo el mesozoico. }\end{array}$ \\
\hline \multirow{6}{*}{ Paleozoico (570-236 MaAP) } & Permiano (282-236 MaAP) & & \multirow{6}{*}{$\begin{array}{l}\text { Grandes variaciones climáticas, el } \\
\text { supercontinente Gondwana en la } \\
\text { latitud sur estaba cubierto de hielo. }\end{array}$} \\
\hline & Carbonífero (346-282 MaAP) & & \\
\hline & Devoniano (402-436 MaAP) & & \\
\hline & Siluriano (435-402 MaAP) & & \\
\hline & Ordovicico (490-435 MaAP) & & \\
\hline & Cámbrico (543-490 MaAP) & & \\
\hline $\begin{array}{c}\text { Precámbrico (Comienzo de la tierra } \\
-570 \mathrm{MaAP})\end{array}$ & & & $\begin{array}{l}\text { Radiación solar } 1 / 3 \text { menor de lo que } \\
\text { es en el presente. Período } \\
\text { extremadamente frio con depósitos } \\
\text { glaciales aún en zonas tropicales. }\end{array}$ \\
\hline
\end{tabular}

Fuente: Elaboración propia a partir de Mokhov y Eliseev (2008). Ma= Millones de años; MaAP=Millones de años antes del presente; $\mathrm{a}=\mathrm{años} ; \mathrm{P}=$ presente; ppmv=partes por millón en volúmen; ppbv=partes por billón en volúmen. 
Al observar este tipo de análisis y en un intento de ser estrictos con la información que se tiene en la actualidad, lo que se observa a través de la historia de la tierra son oscilaciones permanentes en su temperatura pasando de temperaturas bajas a altas y viceversa. Esto contrasta un poco con los resultados de nuestra historia reciente, donde se plantea el hallazgo aparentemente irrefutable de que la acción antropogénica es la que ha generado el mayor impacto sobre el clima de la tierra. No obstante -y este es el dilema- vale la pena preguntarse si es científicamente posible comparar las alteraciones en el clima a partir de los impactos antropogénicos sobre el clima que parecen originarse hace alrededor de 1000 años sobre el clima con otros impactos ajenos a la acción del hombre y que tienen una historia superior a los 500 millones de años. La respuesta por ahora es incierta pero resulta un punto interesante a seguir siendo estudiado por parte de los expertos.

\section{IMPACTOS DEL CAMBIO CLIMÁTICO}

Existe una amplia bibliografía que hace referencia a los impactos del Cambio Climático y dentro de esta, se encuentra una amplia diversidad de aspectos que pueden ser impactados. Dentro de este amplio espectro, una gran cantidad de la bibliografía especializada muestra que los principales impactos del Cambio Climático se dan sobre los recursos costeros, la agricultura, los recursos hídricos, los ecosistemas y la generación y el consumo de energía. Todos estos impactos a su vez afectan el comportamiento de la economía en su conjunto. A continuación se hace una descripción breve alrededor de cada uno de ellos para luego hacer énfasis en los impactos que el Cambio Climático tiene sobre la economía.

Recursos costeros. En cuanto a las costas, en los últimos 100 años el nivel de los océanos se ha elevado debido al calentamiento global y al consecuente deshielo en los glaciares del planeta. Estos a su vez provocan erosiones e inundaciones que generan un deterioro del hábitat así como el desplazamiento de la población costera hacia las zonas continentales (Zanuttigh,2011). En otros casos, en las temporadas secas cuando los ríos disminuyen su caudal se generan intrusiones salinas, lo que causa diversos problemas en términos de la disponibilidad de agua para el consumo humano y la irrigación de productos agrícolas así como el debilitamiento de los suelos en donde se cultiva, afectando variables sociales y económicas en las regiones afectadas.

Agricultura. Los impactos que sobre la agricultura tiene el Cambio Climático son diversos pero pueden clasificarse como directos, indirectos y socioeconómicos. Los primeros son los generados por factores como temperatura, precipitaciones, emisiones de $\mathrm{CO}_{2}$ y eventos climáticos extremos que alteran algunos factores biológicos de los cultivos (ver tabla 2) que modifican positiva o negativamente los patrones de crecimiento de las cosechas (Azzeng et al, 2015), llegando a tener un efecto adverso sobre el crecimiento, la estructura y el ciclo de vida de los cultivos. Los impactos indirectos son los relacionados con la reducción en la calidad y en la cantidad de productos agrícolas y en la fertilidad del suelo así como el surgimiento de plagas, malezas, insectos, enfermedades, etc, mientras que los impactos socioeconómicos están relacionados con el efecto acumulado de los dos anteriores, e incluyen escasez en la oferta de alimentos, perdida de elementos de subsistencia, 
reducción de los ingresos y un incremento en la exposición a vectores de enfermedades. Adicionalmente y desde una perspectiva económica se ven afectados los precios de los productos agrícolas, los retornos esperados hacia los productores y en algunos casos el uso y el valor del suelo.

\section{Tabla 2. Factores climáticos y algunos impactos generales sobre los cultivos}

\begin{tabular}{|c|c|c|c|}
\hline $\begin{array}{l}\text { Variables } \\
\text { climáticas }\end{array}$ & $\begin{array}{l}\text { Tendencias } \\
\text { encontradas }\end{array}$ & $\begin{array}{l}\text { Tendencias } \\
\text { proyectadas }\end{array}$ & $\begin{array}{c}\text { Impacto General sobre el crecimiento } \\
\text { de las cosechas }\end{array}$ \\
\hline \multirow{4}{*}{$\mathrm{CO} 2$} & \multirow{4}{*}{379 ppm en 2005} & \multirow{4}{*}{450 ppm para 2050} & $\begin{array}{l}\text { Incremento neto de la fotosintesis, } \\
\text { producción de biomasa por parte de } \\
\text { las plantas y eficiencia en el uso de la } \\
\text { transpiración }\end{array}$ \\
\hline & & & Transpiración reducida \\
\hline & & & Mayor temperatura de Canopy \\
\hline & & & $\begin{array}{l}\text { Reducciónen la concentración de } \\
\text { nutrientes de las cosechas }\end{array}$ \\
\hline
\end{tabular}

Temperatura
Mínima
En el 2005 se observó un incremento de $0.56^{\circ} \mathrm{C}$ con respecto a 1906

En el 2005 se observó

Promedio un incremento de $0.74^{\circ} \mathrm{C}$ con respecto a 1906

En el 2005 se observó

Máxima un incremento de $0.74{ }^{\circ} \mathrm{C}$ con respecto a 1906

\begin{tabular}{cc} 
& Cambios variables a \\
través del planeta; en & general incremento \\
Lluvias $\quad 0,11 \mathrm{~mm}$. & latitudes a reducción \\
& en latitudes bajas \\
\hline
\end{tabular}

Radiación Reducción en radiación solar e incremento en la Solar fracción de luz difundida
Incremento entre $1.3^{\circ} \mathrm{C}$ y $1.7^{\circ} \mathrm{C}$ en 2050

Incremento entre $1.8^{\circ} \mathrm{C}$ y $4^{\circ} \mathrm{C}$ en 2100
Reducción en el riesgo de escarcha

Incrementos en la conductancia estomática, fotosintesis, respiración y transpiración; crecimiento y desarrollo más rápido, cambios fenológicos; reducción en la eficiencia de la transpiración.

$$
\text { Incremento en el heat stress }
$$

Positivos o negativos, dependiente de la dirección y de otros factores

Incremento en la fotosintesis $\mathrm{y}$ crecimiento debido a la fracción de luz difundida 
Ozono

\begin{tabular}{cccc} 
Troposfera & $50 \mathrm{ppb}$ en el año 2000 & $\begin{array}{c}60-100 \mathrm{ppb} \text { en el año } \\
2050\end{array}$ & $\begin{array}{c}\text { Incremento en el daño foliar, reducción en } \\
\text { el crecimiento y el rendimiento. }\end{array}$ \\
Estratosfera & 265 UD en el año 2000 & $\begin{array}{c}\text { 275-286 UD en el año } \\
\text { 200 }\end{array}$ & $\begin{array}{c}\text { Reducción en la expansión de las hojas y } \\
\text { acumulación de Biomasa }\end{array}$ \\
\hline
\end{tabular}

Fuente: Adaptación del autor a partir de Essang et al (2015).

Recursos hídricos. En este aspecto, los impactos del Cambio Climático que se reflejan sobre la reducción en la disponibilidad de agua generada por cambios en variables como el régimen de lluvias, temperatura, radiación solar y patrones de evaporación-transpiración que afectan el ciclo hidrológico del agua con su consecuente reducción en los glaciares, alterando la disponibilidad de aguas tanto superficiales como los acuíferos, teniendo como consecuencia impactos sobre la agricultura, la salud, los ecosistemas, la biodiversidad y la generación de energía (Kusangaya et al.2013).

Salud. En la literatura los impactos del Cambio Climático sobre la salud son clasificados como directos e indirectos. Los primeros se relacionan con fenómenos generados por alteraciones en variables climáticas como las oleadas de calor, ciclones, inundaciones, tormentas e incendios forestales; los segundos tienen que ver con cambios en el rango, estacionalidad e intensidad en el proceso de transmisión de enfermedades infecciosas, declinación en la cantidad y calidad de agua fresca (Franchini, 2015) así como con la alteración en los patrones de los vectores de transmisión de enfermedades. Entre los impactos más visibles y que afectan principalmente a los países con bajos niveles de desarrollo se destacan la desnutrición en zonas donde se afecta la productividad agrícola y marina; enfermedades cardiopulmonares, asma y alergias, generadas por la producción excesiva de polen de algunas plantas así como enfermedades mentales que surgen a partir de experiencias personales relacionadas con efectos catastróficos y ansiedad dadas las expectativas futuras que genera la ocurrencia de nuevos fenómenos climáticos (Barrett et al.2015).

Energía. De manera similar a lo que sucede con los impactos sobre la agricultura y la salud estos impactos se clasifican en directos o indirectos. En cuanto a los directos, estos tienen que ver con las alteraciones directas a fuentes generadoras de energía donde cambios en variables como el régimen de lluvias, brillo solar, nubosidad y velocidad del viento afectan la generación de energía hídrica, eólica y solar (Burnett et al,2014). Los impactos indirectos están relacionados con la alteración en la cosecha de productos agrícolas que sirven como insumo de los biocombustibles cambios en los patrones de oferta y demanda de energía -principalmente de los hogares- para enfriamiento en zonas que incrementan su temperatura y enfriamiento donde se reduce (Wang and Chen,2014) y cambios importantes en la infraestructura de transmisión de los recursos energéticos, causadas por fenómenos naturales extremos como huracanes que terminan afectando tanto la generación como el consumo de energía. 
Cambio climático: Impactos y perspectivas de investigación desde una visión multidisciplinar

Forero Cantor_Germán Augusto, Saldarriaga Muñoz _ Juan Pablo, Vargas Romero_ Melissa

Ecosistemas. El impacto que genera el Cambio Climático sobre los ecosistemas se puede redefinir mejor en términos de impactos sobre los denominados "servicios" que prestan los ecosistemas ${ }^{6}$ que a su vez pueden ser terrestres o costeros. En este sentido, los impactos terrestres se relacionan con factores como la alteración en el ciclo de las aguas subterráneas (Klove et al, 2014) e impactos biofísicos y socioeconómicos que afectan los sectores agrícola y de silvicultura que se ven reflejados en cambios en la composición y distribución de la vegetación, incremento en la cantidad de incendios forestales, reducción en el ciclo de enfermedades de las plantas y una reducción en la productividad (Leemans and Eickhout, 2004). Los impactos costeros se relacionan principalmente con variación en la acidificación, temperatura y nivel de los océanos, relacionados con la destrucción de manglares y ciénagas que a su vez alteran la distribución de algunas especies animales.

\section{Impactos económicos del Cambio Climático}

Los impactos mencionados con anterioridad guardan amplia interrelación con la economía y en la mayoría de estudios citados se hace énfasis en las pérdidas que se estiman a partir de un análisis costo beneficio para las áreas impactadas. En términos que podríamos denominar puramente económicos las evaluaciones de impacto giran en torno al análisis de riesgos del cambio climático para la economía y a partir de estas sus implicaciones en términos de formulación de la política económica (Stern, 2008). Específicamente, en países como Polonia la caída en el PIB será de $0.046 \%$ y en Austria del $0.03 \%$ para los próximos 50 años, explicado este cambio principalmente por la alteración en el nivel de los océanos (Bosello et al, 2011). En cuanto a los impactos totales del cambio climático se encuentran efectos positivos y negativos del PIB siendo estos extremos negativamente en países como Timor Oriental (-19\%) y positivos en China (6\%) (Tol, 2010, 2012).

Medición. Uno de los puntos de la discusión central alrededor de los impactos del Cambio Climático ha sido su metodología de medición. Inicialmente las metodologías utilizadas eran limitadas y por ende sus aproximaciones eran muy pobres, explicándose esto por la ausencia de la influencia que podrían tener los diversos mecanismos de adaptación al Cambio Climático al interior de los modelos que se planteaban lo cual llevaba a una sobreestimación de los impactos del Cambio Climático (Nordhaus, 1993).

La evolución de las metodologías de medición ha llevado a que se planteen distintos enfoques de medición que se pueden resumir en dos: el enumerativo, que hace referencia principalmente a los efectos físicos del cambio climático, los cuales son establecidos a partir de las ciencias naturales y luego son valorados a precios de mercado, con una limitación que es la extrapolación que se hace a partir de sus estimaciones. Este tipo de análisis es considerado como del tipo costo-beneficio y se ha concentrado principalmente en valorar impactos del clima sobre la agricultura, la salud y la infraestructura. El otro planteamiento es el que se puede denominar de aproximación estadística, que se enfoca en estimar los impactos del clima sobre la actividad y el bienestar económicos, utilizando técnicas de

6 Estos servicios se pueden definir como los procesos por los cuales los ecosistemas generan recursos necesarios para la vida humana como el agua, madera, estructuras apropiadas para la pesca, polinización de plantas, etc. 
econometría y estadística espacial a través de las regiones dentro de los países y en la mayoría de los casos utilizando agregaciones para establecer los impactos del Cambio Climático. Este método está basado en alteraciones en los niveles de ingreso relacionadas con cambios en el clima más que con diferencias extrapoladas en las dos variables; sin embargo su limitación está en que en la mayoría de los casos se omite el efecto de algunas variables de medición importantes generando sesgos en las estimaciones.

De otro lado se encuentran los modelos del tipo "Top-Down" y "Bottom-Up" (Mendelson et al, 2000) que son los que en los últimos años han sido utilizados para valoración de impactos del Cambio Climático. Los primeros, toman como base la demanda agregada y calculan los daños globales como una función de los cambios en la temperatura media global; su debilidad radica en que carecen de una estructura espacial y estructural. Los modelos "Bottom-up" capturan efectos directos individuales dados por los modelos climáticos de manera espacial, sin embargo son débiles en el sentido que no hacen estimaciones de daño y no incorporan mecanismos de adaptación y requieren una alta capacidad tecnológica de procesamiento de los datos.

Finalmente, los modelos más populares para la medición de impactos del Cambio Climático son los Modelos de Valoración Integrada (IAM por sus siglas en inglés). Estos son modelos que incluyen el clima y otros aspectos relacionados con las ciencias puras y la economía del cambio climático. Estos evalúan las emisiones y el cambio climático generados por el crecimiento económico, estableciendo luego los impactos sobre la economía y proyectando los resultados de las posibles políticas de mitigación del Cambio Climático. La ventaja de estos modelos es que representan todos los procesos de principio a fin, siendo la principal desventaja que simplifican algunos de los procesos que son analizados con más detalles en modelos más completos (Pindyck,2013). La tabla 3 muestra en forma general cuales son los componentes de este tipo de modelos.

Tabla 3. Principales componentes de los modelos de valoración integrada (IAM)

\begin{tabular}{|l|l|}
\hline \multicolumn{1}{|c|}{ Elemento } & \multicolumn{1}{c|}{ Algunas características } \\
\hline $\begin{array}{l}\text { Proyecciones de emisiones } \\
\text { futuras de } \mathrm{CO}_{2}\end{array}$ & $\begin{array}{l}\text { Requieren proyecciones del crecimiento del PIB y la intensidad } \\
\text { del carbono asociada al mismo bajo lo que se denomina un } \\
\text { escenario ideal de los negocios (BAU) }\end{array}$ \\
\hline $\begin{array}{l}\text { Proyecciones de } \\
\text { concentraciones de } \mathrm{CO}_{2} \text { en la } \\
\text { atmósfera. }\end{array}$ & $\begin{array}{l}\text { Se estiman a partir de las emisiones pasadas, presentes y } \\
\text { futuras de } \mathrm{CO}_{2} .\end{array}$ \\
\hline $\begin{array}{l}\text { Proyecciones de cambios en } \\
\text { la temperatura global y otras } \\
\text { variables climáticas. }\end{array}$ & $\begin{array}{l}\text { Estas estimaciones se realizan a partir de la concentración } \\
\text { establecida de } \mathrm{CO}_{2} .\end{array}$ \\
\hline $\begin{array}{l}\text { Proyecciones de impacto } \\
\text { económico }\end{array}$ & $\begin{array}{l}\text { Se expresan en términos de pérdidas de PIB y consumo } \\
\text { generadas a partir de grandes variaciones en las temperaturas. } \\
\text { Adicionalmente se valoran otros efectos adversos de carácter } \\
\text { social, político y de salud que son monetizados y se incluyen } \\
\text { como parte del PIB. }\end{array}$ \\
\hline
\end{tabular}


Cambio climático: Impactos y perspectivas de investigación desde una visión multidisciplinar

Forero Cantor__Germán Augusto, Saldarriaga Muñoz _ Juan Pablo, Vargas Romero _ Melissa

\begin{tabular}{|l|l|}
\hline $\begin{array}{l}\text { Estimación de los costos de } \\
\text { reducción }\end{array}$ & $\begin{array}{l}\text { Se establecen los montos necesarios para mitigar los efectos } \\
\text { generados por cambios actuales y futuros en el clima. Se } \\
\text { involucran mejoras tecnológicas que pueden reducir los costos } \\
\text { de reducción futuros. }\end{array}$ \\
\hline $\begin{array}{l}\text { Utilidad social y tasa de } \\
\text { preferencia intertemporal }\end{array}$ & $\begin{array}{l}\text { Se tienen en cuenta los costos de reducción que a su vez } \\
\text { generan pérdidas en el consumo y se valoran con respecto a } \\
\text { ganancias en consumo futuro generado por reducciones en el } \\
\text { calentamiento global. }\end{array}$ \\
\hline
\end{tabular}

Fuente: Elaboración propia a partir de Pindyck (2013).

Aunque este tipo de modelos son de amplia difusión no dejan de generar controversia dados los diversos resultados que se pueden llegar a obtener para un mismo contexto. Dicha controversia puede ser zanjada por el hecho de que los investigadores pueden realizar diferentes tipos de modelación de manera arbitraria, donde cada uno elige formas funcionales, valores de los parámetros y de otros insumos de manera individual, que afectan directamente el valor de los impactos y sus costos de reducción.

Impactos encontrados por los modelos. En general la valoración de los impactos se ha concentrado en establecer las pérdidas para la economía en términos de PIB tanto a nivel global como sectorial. Un primer gran hallazgo es que los impactos generados por el Cambio Climático pueden impactar tanto como positiva como negativamente algunas regiones del mundo. En este sentido, los impactos positivos más importantes se concentran sobre algunos países de Europa del este y la antigua Unión Soviética, mientras que los impactos negativos más fuertes se dan sobre Suramérica, África y China. Otros análisis (Mendelson et al, 2000) incluyen otros perdedores como Oceanía y Asía así como otros ganadores como otros países de Europa y los Estados Unidos encontrando un efecto neto positivo del 0.3\% del PIB. Desde esta perspectiva los beneficios del cambio climático se explican debido a que la mayor concentración de la producción mundial se encuentra en las zonas templadas y un leve incremento en la temperatura genera efectos positivos en términos de reducción de costos de calentamiento y de enfermedades relacionadas con los climas frios.

Aunque la mayor parte de la población está concentrada en los trópicos el impacto económico no es muy fuerte porque la concentración de la producción no alcanza las mismas proporciones de la población y es en este sentido que las ganancias en regiones por fuera del trópico contrarrestan las pérdidas que allí se generan. Si bien esto es cierto, surge una inquietud y es que aunque los beneficios netos son mayores gracias a los países de alto ingreso, es en estos donde las emisiones de gases de invernadero son mayores y los impactos de dichos gases de invernadero son mayores en los países de bajo ingreso.

Otras estimaciones de los impactos son más catastróficas. Stern (2007) plantea que en un escenario "optimista" sin medidas para mitigar el Cambio Climático se tendrían pérdidas de al menos un 5\% del PIB cada año durante un período de tiempo indefinido y si se tuviesen en cuenta de manera detallada otros riesgos e impactos está cifra podría alcanzar un valor del 20\% ó más. Otros análisis establecen la caída en el PIB a partir de una reducción en 
la productividad laboral, la cual explica el 84\% de dicha reducción y estimando para esta variable una caída en el año 2050 del 1.8\% a nivel global (Roson and van der Mensbrugghe, 2010).

\section{El Cambio climático en Colombia}

Aunque la problemática del Cambio Climático no es reciente y está afectando principalmente a los países que se encuentran ubicados en el trópico, Colombia parece haber sido ajena al problema. Al hacer una revisión de la literatura, los trabajos académicos aunque importantes aún son aislados, destacándose sin embargo el amplio y creciente interés que ha comenzado a despertar el tema. En términos de política pública, esta pareciera tener la misma dinámica ya que los esfuerzos se han concentrado principalmente en establecer estrategias de corto plazo para la prevención ante los desastres sociales generados por alteraciones en el clima (CEAH, 2010) dejando de lado estrategias de adaptación y mitigación en el mediano y largo plazo para los impactos del Cambio Climático.

Impactos del Cambio Climático en Colombia. Al analizar los impactos, no existen aún para Colombia estudios detallados como los que se tienen a nivel internacional. Un primer elemento a tener en cuenta cuando se analizan los impactos es la geografía y en este sentido los mayores impactos se presentan en las regiones de mayor altura sobre el nivel del mar que es donde se concentra la mayoría de la población colombiana y que es donde ante el Cambio Climático se da una mayor escasez hídrica e inestabilidad de los suelos mientras que en las costas como proporción de la población el impacto es menor.

Dentro de los mayores impactos se destaca la alteración regional del régimen de lluvias, el cual se ha alternado, incrementándose en zonas donde históricamente había sido bajo y viceversa, se reduce en zonas que históricamente mostraban un régimen alto. En este sentido el principal impacto han sido las inundaciones y sequías que alteran la disponibilidad de agua potable y en algunos casos la generación de energía eléctrica (Mance y Rodriguez, 2009), afectando asentamientos humanos y el desempeño de algunas actividades económicas claves para las regiones.

De otra parte, otro impacto encontrado se da sobre los glaciares y el nivel del mar. En este sentido, se ha presentado una reducción de entre 50 y $100 \mathrm{~cm}$. de espesor por año para los primeros y en los últimos 10 años el nivel del mar se ha incrementado a una tasa de 3.1 milimetros por año. Específicamente, en ciudades como Cartagena y Tumaco se han establecido incrementos de $5 \mathrm{~mm}$. al año durante los últimos 50 años, $10 \mathrm{~cm}$. en la región caribe y $22 \mathrm{~cm}$. en la pacífica; adicionalmente, la temperatura en los últimos 50 años se ha incrementado hasta $0.65^{\circ} \mathrm{C}$, siendo más alta a mayores alturas. Para el período (1971-2000) el incremento en la temperatura se ha establecido en $0.13^{\circ} \mathrm{C}$, esperando que dicho incremento alcance $1.4^{\circ} \mathrm{C}$ para el período $2011-2040,2.3^{\circ} \mathrm{C}$ del $2041 \mathrm{al} 2070$ y $3.2^{\circ} \mathrm{C}$ del 2071 hasta el 2100, siendo afectados principalmente los departamentos de Norte de Santander, Risaralda, Huila, Sucre y Tolima (CEPAL, 2013). 
Cambio climático: Impactos y perspectivas de investigación desde una visión multidisciplinar

Forero Cantor_Germán Augusto, Saldarriaga Muñoz _ Juan Pablo, Vargas Romero_ Melissa

En términos puramente económicos y en una primera aproximación formal a los impactos económicos del Cambio Climático (DNP-BID,2014) se establece un escenario de medición de impacto sobre el producto, en el que se contrarrestan efectos positivos y negativos del Cambio Climático sobre las diferentes ramas de actividad económica, encontrando que las pérdidas anuales por el cambio climático para el período 2011-1100 estarían alrededor de $0.49 \%$ del PIB por cada año.

Ante este panorama, es claro que en Colombia las investigaciones relacionadas con los impactos generados por el Cambio Climático ya sean estos económicos o de otra índole son incipientes y generan más preguntas que respuestas, generando una amplia ventana de necesidades y oportunidades para que desde el sector académico tanto estudiantes como profesionales de distintas disciplinas comiencen a desarrollar investigaciones que respondan a ellas.

\section{CONCLUSIONES}

El Cambio Climático llegó para quedarse y cada día sus impactos sociales y económicos se perciben con mayor intensidad no solamente en los países desarrollados sino en todo el planeta lo que lo ha convertido en un fenómeno de interés global. Sin embargo, en países como Colombia no existe aún un consenso general en cuanto a la necesidad de analizar los impactos que está generando el Cambio Climático sobre aspectos como la reducción de los recursos costeros, la productividad agrícola, la disponibilidad de recursos hídricos, el comportamiento de los ecosistemas, la generación y el consumo de energía la producción agrícola y la salud -solo por mencionar algunos- que están afectando de manera directa a la sociedad colombiana en su conjunto. Es interesante mencionar a su vez que a nivel mundial los estudios relacionados tanto con el Cambio Climático como sus impactos tienen aún un desarrollo no consensuado en términos metodológicos ya que en la mayoría de los casos son abordados desde distintas aproximaciones teóricas lo que lleva a su vez a que sus resultados sean diversos y no exista aún unidad de criterio en cuanto a los resultados obtenidos en sus estimaciones.

Es entonces el Cambio Climático un fenómeno que dadas sus múltiples dimensiones puede llegar a ser una fuente inagotable de ideas para el desarrollo de investigaciones multidisciplinarias en donde de manera específica pueden jugar un papel muy importante el aporte de las distintas disciplinas enmarcadas dentro de las Ciencias Económicas y Administrativas. Es por esto que tomando como marco de referencia el contexto del presente artículo se podría pensar desde la academia en promover el desarrollo de estudios rigurosos relacionados tanto con los impactos económicos del Cambio Climático sobre variables sociales y económicas que afectan el comportamiento económico regional y nacional sino ser propositivos en otros temas de investigación relacionados con el Cambio Climático tales como el diseño de mecanismos de adaptación y mitigación ante los impactos y el desarrollo de modelos de gestión de riesgo ante la presencia del Cambio Climático, esto sólo por mencionar algunas. Relacionado con esto y más importante aún es involucrarse en el planteamiento y desarrollo de nuevas metodologías de valoración, las 
cuales aún son casi que inexistentes en Colombia y son una fuente inagotable de estudio. Es una tarea interesante y estamos a tiempo de comenzarla.

\section{REFERENCIAS}

(1) Asseng, S., Zhuy, Y., Wang, E. and Zhang, W., (2015). Crop modeling for climate change impact and adaptation, in Sadras, V and Calderini, D. (Eds), CROP PHYSIOLOGY, APPLICATIONS FOR GENETIC IMPROVEMENT AND AGRONOMY, (second edition), pp.505-546, New York, USA: Elsevier.

(2) Barrett, B., Charles, J. and Temte, J. (2015). Climate Change, human health, and epidemiological transition. Preventive Medicine, 70, 69-75.

(3) Bosello, F., Nicholls, R, Richards, J., Roson, R., and Tol, R. (2011). Economic impacts of Climate Change in Europe: Sea Level Rise. Climate Change, 112, 63-81.

(4) Bradley, R. (2015). Paleoclimatology: Reconstructing climates from the Quaternary (third edition), Oxford, UK: Elsevier.

(5) Burnett,D., Barbour, E. and Harrison, G. (2014). The UK solar energy resource and the impact of climate change. Renewable Energy, 71, 333-343.

(6) Burroughs, W. (2007). Climate Change A Multidisciplinary Approach (Second Edition), Cambridge, UK: Cambridge University Press.

(7) CEAH, 2010. Estado actual, perspectivas y prioridades para los preparativos ante desastres en Colombia, Documento país -DP 2010. Comisión Europea de Ayuda Humanitaria, Bogotá.

(8) CEPAL (2013). Panorama del Cambio Climático en Colombia, Serie Medio Ambiente y Desarrollo,Santiago de Chile, 146 .

(9) DNP-BID (2014). Impactos Económicos del Cambio Climático en Colombia- Síntesis-. Bogotá, Colombia.

(10) Fleming, J. (1998), Historical Perspectives On Climate Change, New York, USA: Oxford University Press.

(11) Franchini, M. and Mannucci, M.(2015).Impact on Human Health of climate changes, European journal of Internal Medicine, 26, 1-5.

(12) Klove, B., Ala-Aho, P., Bertrand, G., Gurdak, J., Kupfersberger, H., Kvaerner, J., Muotka,T., Mykra, H., Preda, E. Rossi, P., Uvo, C., Velasco, E., Pulido-Velasquez, M. (2014). Climate change impacts on groundwater and dependent ecosystems, Journal of Hidrology, 518, 250-266.

(13) Kusangaya, S., Warburton, L., van Garderen, E. and Jewitt, G. (2013), Impacts of climate change on water resources in southern Africa: A review. Physics and Chemistry of the Earth, 67, 47-54.

(14) Leemans, R. and Eickhout, B. (2004). Another reason for concern: regional and global impacts on ecosystems for different levels of climate changes. Global Environmental Change, 14, 219-228.

(15) Letcher, T. (2009), Climate Change: Observed Impacts on Planet Earth. Amsterdan, Netherland: Elsevier. 
(16) Mance, H. y Rodriguez, M. (2009). Cambio Climático: lo que está en juego. Bogotá, Colombia. Universidad de los Andes, FESCOL, WWF, Fundación Natura, Ecofondo, Fundación Alejandro Angel Escobar, Tropenbos International.

(17) Mendelson, R., Morrison, W., Schlesinger, M. and Andronova, N. (2000) COUNTRY SPECIFIC MARKET IMPACTS OF CLIMATE CHANGE. Climatic Change, 45, 553-569.

(18) Mokhov, I. and Eliseev, A. (2008), Climate Change 3: History and Current State. in Encyclopedia of Ecology, pp.598-602, Oxford, UK: Elsevier.

(19) Naciones Unidas, (1992). Convención Marco de las Naciones Unidas sobre el Cambio Climático. New York, USA: UN.

(20) Nordhaus, W. (1993), Reflections on the Economics of Climate Change, The Journal of Economic Perspectives, 7 (4), 11-25.

(21) Pindyck, R. (2013), Climate Change Policy: What do the model tell us?, Journal of Economic Literature 51, $3,860-872$.

(22) Roson, R. and van der Mensbrugghe, D. (2010), Climate Change and Economic Growth: Impact and interactions. Working paper No. 07, Department of Economics, University of Venice.

(23) St. Louis, M. and Hess, J. (2008), Climate Change, Impacts on and Implications for Global Health, American Journal of preventive Medicine, 35(5), 527-538.

(24) Stern, N. (2008), The Economics of Climate Change, American Economic Review: Papers and proceedings, 98 (2), 1-37.

(25) Stern, N. (2007). The Economics of Climate Change The Stern Review, Cambridge, UK: Cambridge University Press.

(26) Toan, Q. (2014), Climate Change and Sea Level Rise in the Mekong Delta: Flood, Tidal Inundation, Salinity Intrusion, and Irrigation Adaptation Methods. In Thao, D.,Takagi, H. and Esteban, M. (Eds.) Coastal Disasters and Climate Change in Vietnam, pp.199-218. Oxford, UK: Elsevier.

(27) Tol, R. (2013), The economic impact of climate change in the 20th and 21st centuries. Climate Change, 117, 795-808.

(28) Tol, R. (2010), The Economic Impact of Climate change, Perspektiven der Wirtschaftspolitik, 11(s1):13-37.

(29) Wang, H. and Chen, Q. (2014). Impact of climate change heating and cooling energy use in buildings in the United States.

(30) Weart, S.(2015), History of Scientific Work on Climate Change, Reference Module in Earth Systems and Environmental Sciences, pp.87-89, Oxford, UK: Elsevier.

(31) Zanuttigh, B. (2011), Coastal Flood protection: What perspective in a changing climate? The THESEUS approach. Environmental Science \& Policy, 14, 845-863. 